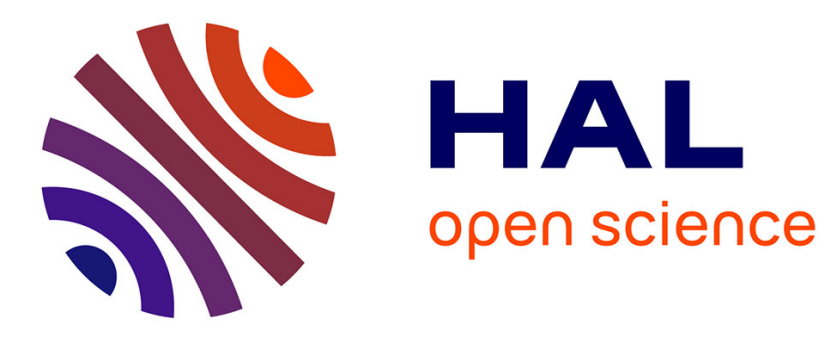

\title{
Statistical shape model to generate a planning library for cervical adaptive radiotherapy
}

Bastien Rigaud, Antoine Simon, Maxime Gobeli, Julie Leseur, Loig Duverge, Daniele Williaume, Joël Castelli, Caroline Lafond, Oscar Acosta, Pascal Haigron, et al.

\section{To cite this version:}

Bastien Rigaud, Antoine Simon, Maxime Gobeli, Julie Leseur, Loig Duverge, et al.. Statistical shape model to generate a planning library for cervical adaptive radiotherapy. IEEE Transactions on Medical Imaging, 2019, 38 (2), pp.406-416. 10.1109/TMI.2018.2865547 . hal-01988100

\section{HAL Id: hal-01988100 https://hal-univ-rennes1.archives-ouvertes.fr/hal-01988100}

Submitted on 22 Mar 2019

HAL is a multi-disciplinary open access archive for the deposit and dissemination of scientific research documents, whether they are published or not. The documents may come from teaching and research institutions in France or abroad, or from public or private research centers.
L'archive ouverte pluridisciplinaire HAL, est destinée au dépôt et à la diffusion de documents scientifiques de niveau recherche, publiés ou non, émanant des établissements d'enseignement et de recherche français ou étrangers, des laboratoires publics ou privés. 


\title{
Statistical shape model to generate a planning library for cervical adaptive radiotherapy
}

\author{
Bastien Rigaud $M S c^{*}$, Antoine Simon $P h D$, Maxime Gobeli, $M D$, Julie Leseur, $M D$, Loig Duvergé, \\ $M D$, Danièle Williaume, $M D$, Joël Castelli $M D, M S c$, Caroline Lafond $P h D$, Oscar Acosta $P h D$, \\ Pascal Haigron $P h D$, Renaud De Crevoisier $M D, P h D^{*}$
}

\begin{abstract}
External beam radiotherapy is extensively used to treat cervical carcinomas. A single planning CT scan enables the calculation of the dose distribution. The treatment is delivered over 5 weeks. Large per-treatment anatomical variations may hamper the dose delivery, with the potential of an organs at risk (OAR) overdose and a tumor underdose. To anticipate these deformations, a recent approach proposed three planning CTs with variable bladder volumes, which had the limitation of not covering all per-treatment anatomical variations. An original patient-specific population-based library has been proposed. It consisted of generating two representative anatomies, in addition to the standard planning $C T$ anatomy. First, the cervix and bladder meshes of a population of 20 patients (314 images) were registered to an anatomical template, using a deformable mesh registration. An iterative point-matching algorithm was developed based on local shape context (histogram of polar or cylindrical coordinates and geodesic distance to the base) and on a topology constraint filter. Second, a standard principal component analysis (PCA) model of the cervix and bladder was generated to extract the dominant deformation modes. Finally, specific deformations were obtained using posterior PCA models, with a constraint representing the top of the uterus deformation. For a new patient, the cervix-uterus and bladder were registered to the template, and the patient's modeled planning library was built according to the model deformations. This method was applied following a leaveone-patient-out cross-validation. The performances of the modeled library were compared to those of the three-CT-based library and showing an improvement in both target coverage and OAR sparing.
\end{abstract}

Index Terms-radiotherapy, cervical, deformable mesh registration, PCA model, planning library

\section{INTRODUCTION}

$\mathrm{E}$ XTERNAL beam radiotherapy (EBRT) with chemotherapy, followed by brachytherapy, is the reference treatment for locally advanced cervical carcinomas. EBRT is based on the acquisition of a single planning CT scan on which anatomical structures are manually delineated and from which the dose distribution is calculated. The clinical target volume (CTV) is defined as the cervical tumor, uterus, parametria and vagina according to initial staging, and pelvic lymph nodes. A planning target volume (PTV) is defined, corresponding to the CTV, with a fixed margin (generally of $10 \mathrm{~mm}$ ). The organs at risk (OAR) are the bladder, rectum, and bowel, with the latter

Acknowledgments: This work was partly funded by the French National Research Association (ANR) as part of the Investissement d'Avenir program (Labex CAMI) under reference ANR-11-LABX-0004, by the French "Ligue contre le Cancer", and by the French organization "La Vannetaise". including the sigmoid. The dose distribution is then optimized to deliver the prescribed dose to the PTV, while sparing as much as possible of the OAR. The treatment is delivered in daily fractions, over 5 weeks. On-board imaging, such as cone-beam CT (CBCT), enables the visualization of anatomical structures (cf. Fig. 1a). However, large anatomical variations may occur over treatment, such as bladder and rectum filling or tumor regression. The position of the uterus has been shown to be related to bladder volume: an empty bladder results in an anteriorly tipped uterus and a full bladder results in a vertical uterus $[1,2]$. These deformations may, consequently, hamper the precision of the dose delivery since the per-treatment anatomy does not correspond to the planning anatomy ( $c f$. Fig. 2 ). The patient may be exposed to both tumor underdose and OAR overdose [3-5].

Several strategies have previously been proposed to account for these anatomical variations. The simplest solution is to increase the size of the PTV by increasing the CTV-to-PTV margin. Another option is to define an internal target volume (ITV) that corresponds to the union of different CTVs from planning CT acquired with various bladder volumes. These two solutions limit the risk of CTV underdosage, yet carry the major drawback of increasing the dose delivered to the OAR [6-8]. Thus, recently, more sophisticated strategies, known as adaptive radiation therapy (ART), have been proposed. ART considers not only one treatment plan but multiple treatment plans, adapted to different anatomical configurations that anticipate per-treatment organ deformations. In EBRT for cervical cancer, the main ART strategy relies on the generation of a treatment planning library $[7,8]$. This treatment planning library is composed of multiple treatment plans based on different anatomies (i.e., CTV shapes) resulting from planning CTs acquired with different bladder volumes ( $c f$. Fig. 1b). The "classic" CT-based library is generally based on two or three anatomies (empty, intermediate and full bladder volume) in order to provide adequate coverage, while limiting the workload of the treatment plan optimization (one for each CT). At each treatment fraction, CBCT images enable the optimal treatment plan to be chosen among those of the library ("planof-the-day"). This strategy appears to be adequate to compensate for uterine motions [9-11]. However, such a library

All authors are with Univ Rennes, CLCC Eugène Marquis, Inserm, LTSI UMR 1099, F-35000 Rennes, France.

* Corresponding authors; email address: bastien.rig@gmail.com; r.decrevoisier@rennes.unicancer.fr, tel.: +33(0)299253031 


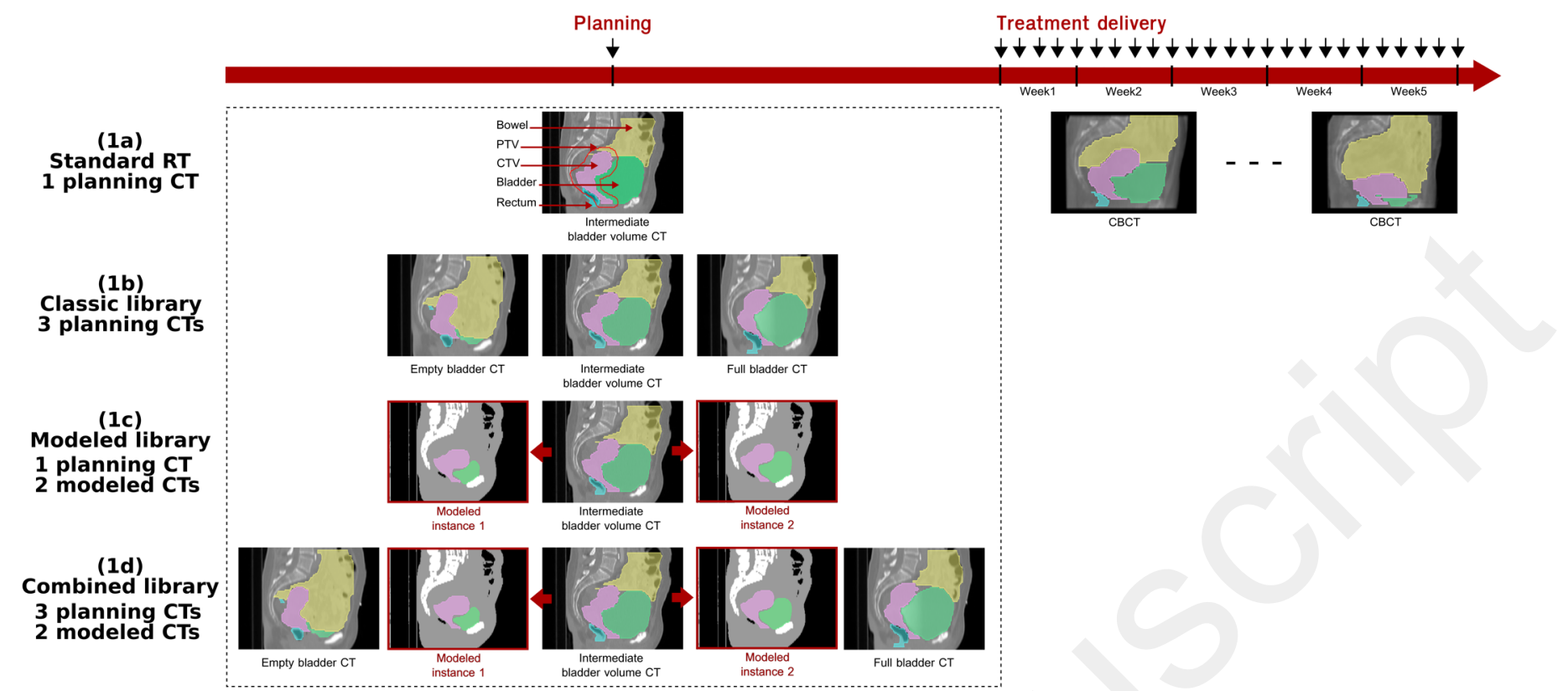

Fig. 1. Radiotherapy strategies for locally advanced cervical cancer

In the standard RT approach (1a), the dose distribution is calculated based on one planning CT, generally with intermediate bladder volume, and the treatment is delivered in $25^{\text {th }}$ fractions over 5 weeks. However, large per-treatment anatomical deformations, mainly due to the bladder filling and tumor shrinking, can be observed. These deformations may not be taken into account on the single planning CT, thereby exposing the patient to tumor underdose and organ at risk overdose. Three alternative strategies are represented: (1b) a classic library-based RT with three planning CTs (variable bladder volumes); (1c) the proposed modeled planning library based on the population analysis; and (1d) the combined library using (1b) and (1c).

RT: Radiation therapy; PTV: Planning target volume; CTV: Clinical target volume

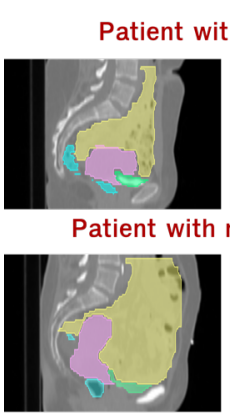

Empty bladder CT
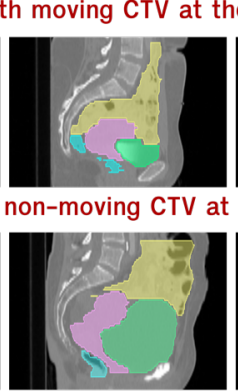

Intermediate bladder volume $\mathrm{C}$
Per-treatment deformations

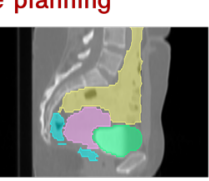

the planning

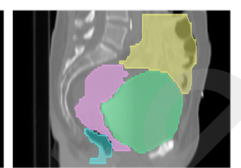

Full bladder CT
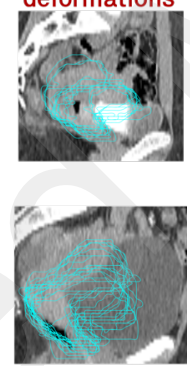

Fig. 2. Classic CT-based planning library limitations

Two patients are illustrated. One patient had a moving CTV at the planning and during the treatment. The classic library appears to be adapted. Another patient had a non-moving CTV at the planning and a moving CTV during treatment, resulting in tumor underdose and OAR overdose when using the classic library.

The CTV (cervix-uterus) is in pink. The rectum, bladder and bowel are in blue, green and yellow, respectively. The per-treatment CTV contours are in light blue. CTV: Clinical target volume; OAR: Organ at risk

does not handle per-treatment deformations that are not represented in the planning CTs. In particular, this strategy is inefficient for patients with no motion at the planning and motion during treatment ( $c f$. Fig. 2) [8]. Furthermore, compared to the standard treatment, the workload required to acquire and delineate the CT images is relatively heavy (i.e., 1 hour instead of 20 minutes for the whole acquisition procedure, and 1 hour 30 minutes instead of 30 minutes for manual segmentation) while causing more discomfort to the patient (i.e., bladder filling protocol).

One way to overcome these limitations would be to simulate the most likely deformations of the patient's anatomy in order to improve the coverage of the target. For this purpose, a

population analysis can be used to identify the main deformations. This population analysis needs the cervix-uterus of the different patients to be registered. This registration step is challenging due to the large intra-patient and all the more inter-patient variations. To our knowledge, only one study analyzed both intra and inter-patient deformations of the cervixuterus anatomy in the context of EBRT [12]. Based on CT images, the proposed approach used deformable registration and principal component analysis (PCA) to quantify the delivered dose uncertainties.

The aim of this study was, based on a population analysis, to generate a patient-specific planning library (CTV and bladder) using a single CT scan of the considered patient ( $c f$. Fig. 1c). First, a deformable mesh registration (DMR) method was used to quantify the population deformations within a template space. This DMR was based on the shape context descriptors using an iterative and multiscale approach. Second, standard and posterior principal component analysis (sPCA and pPCA) were used to build a statistical shape model (SSM) in the template space. By using different motion constraints associated with the top of the uterus, deformation vector fields (DVF) between the mean sPCA and pPCA models were generated. Finally, the DVFs were used to deform the patient CTV and bladder, thereby, generating the different shapes of the patient-specific library.

This paper first describes the proposed workflow, including the DMR method, the sPCA and pPCA approaches and the method used to model a patient-specific library. This approach was then evaluated by comparing it with the standard RT and a three-CT-based planning library (i.e., "classic" library). The endpoints were the geometric coverages of the CTV and OAR by the PTV throughout the whole treatment. 


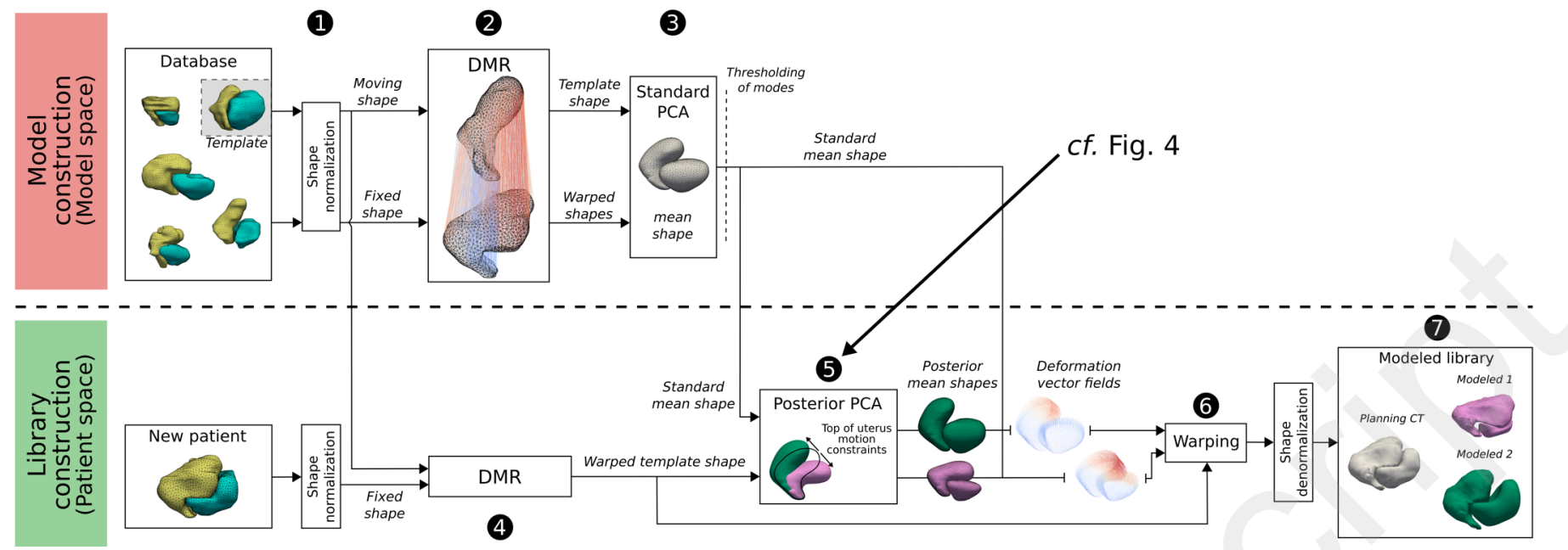

Fig. 3. Model workflow used to generate the modeled library

The first step is the model construction: (1) the organ shapes are normalized to limit the observations to the uterus movement; (2) the DMR method is used to compute the correspondence fields between the template and the population shapes; and (3) the standard PCA is built over the shapes in correspondences to represent the dominant deformations.

The second step is the library construction to apply the model to a new patient: (4) the organ shapes are propagated in the model space by means of the DMR method; (5) a posterior PCA is built to extract the desired deformation corresponding to the top of the uterus deformation; (6) the shapes are then warped by the deformation vector fields of the model; and (7) the warped shapes are denormalized to define the modeled planning library in the patient space.

DMR: deformable mesh registration; PCA: principal component analysis

\section{METHODS}

\section{A. Proposed Workflow}

The workflow of the model construction is presented in Fig. 3. The first step was the population model construction. First, the meshes of the cervix-uterus (i.e., CTV) and the bladders of the patient population were aligned and normalized according to an anatomical template. Second, DVF between the template and the inter- and intra-patient shapes were obtained using DMR. Third, the SPCA was built over these shapes in correspondence to generate a SSM.

Then, for a new patient's shapes (CTV and bladder), normalization and DMR correspondences with the anatomical template were computed. According to the CTV shape of the new patient, motion constraints were generated over the top of the uterus of the sPCA mean shape in order to obtain the pPCA models.

Finally, the new patient planning shapes (CTV and bladder) were deformed by means of the DVF between the standard and posterior mean shapes. This enabled the definition of several instances around the patient CTV and bladder shapes, which formed the planning library.

Each of these steps is described here-after.

\section{B. Template Selection and Shape Normalization}

A template shape $\left(\mathrm{S}_{\text {temp }}\right)$ was selected as the CTV, having the median volume of the database. To analyze the patients in a common space (i.e., scale and position), a shape normalization was first applied:

$S_{\text {norm }}(x, y, z)=$

$\underline{\max \left\{\operatorname{GeoD}\left[S_{\text {temp }}(x, y, z)\right]\right\} \times\left[S(x, y, z)-C_{\text {centroid }}(x, y, z)\right]}$

$\max \{\operatorname{GeoD}[S(x, y, z)]\}$
Each patient's CTV shapes, $S(x, y, z)$, were size- and position-normalized. The size-normalization was based on the maximum of the geodesic distance $(G e o D)$ to the base centroid of the planning CTV. The position-normalization was related to the CTV base centroid coordinates $\left(C_{\text {centroid }}(x, y, z)\right)$. Finally, each patient's CTV shapes were size-normalized by the maximum GeoD of the template CTV. The maximum of the $G e o D$ to the cervix centroid was also used to identify the position of the top of the uterus (ToU). The bladder shapes were normalized using the same transformations.

\section{Iterative Deformable Mesh Registration Based on Shape Context}

To quantify the population deformations, point-to-point correspondences of organ shapes had to be estimated. Shapes can be represented in a common domain using skeletal representation [13] or spherical harmonic decomposition [14, 15], which can be limited for complex shapes.

More commonly, DMR methods have been introduced to estimate a DVF between two meshes. The DVF is generally estimated using local correspondences estimated by morphological descriptors. Shape context descriptors have been shown to be efficient for this purpose [16]. This method has been enriched with a depth feature [17] or filtered by a geodesic distance-based topology criterion [18] to improve its robustness. However, DMR based on a shape context descriptor still remains highly sensitive to point mismatch when computed in one step. Filtering and regularization may, therefore, be required to constrain the transformation and to ensure more realistic behavior [19]. Iterative DMR methods have been developed, such as surface-based algorithms, thin plate spline robust-point matching (TPS-RPM) [20-23] and coherent point drift (CPD) [24]. Nevertheless, these algorithms rely on pointto-point distances to drive the DVF. They are, thus, dependent 
on the alignment of the shapes and ultimately may lead to uncertainties in inter-patient soft tissue matching when large deformations have to be estimated, without complex parametrization.

The proposed DMR aims to estimate the forward DVF, representing the elastic transformation $T$ between the fixed (i.e., the template) and the moving shapes $\left(S_{\text {fixed }}, S_{\text {moving }}\right)$. The considered shapes are the cervix-uterus and the bladder, which are highly deformable organs. This iterative process is expressed by the following equations:

$D V F=D V F+G\left(\mu \times D V F_{\text {step }}\right)$

$S_{\text {warped }}=T\left(S_{\text {moving }}, D V F\right)$

At each iteration, the $S_{\text {moving }}$ shape is warped ( $\left.S_{\text {warped }}\right)$ by the $D V F$, which is updated by means of the $D V F_{\text {step }}$. The $D V F_{\text {step }}$ is computed, at each iteration, between $S_{\text {fixed }}$ and $S_{\text {warped }}$ using a shape context descriptor [16] and the normalized geodesic distance to the centroid of the organ base, as described below. $D V F_{\text {step }}$ is then interpolated and regularized by a thin plate spline (TPS) transformation and a Gaussian kernel $(G)$, respectively, and is weighted by $\mu \in] 0,1](\mu=1$ at the last iteration).

The descriptors are calculated on $S_{\text {fixed }}$ and $S_{\text {moving, with each }}$ shape being represented by $N$ points. For each point $p_{i}$, a normalized histogram, $h_{i}$, is defined, representing the spherical (resp. cylindrical) coordinate difference between this point and the remaining $N-1$ points of the shape. Thus, for each point of the bladder (resp. CTV), $\beta \times \gamma \times \delta=N b i n s$, histogram bins are defined, with $\beta=5$ for the radial distance (resp. $\mathrm{x}$-y distance), $\gamma=12$ for the azimuth angle and $\delta=6$ for the elevation angle (resp. $z$ signed distance) with a range of $[0,100],[-180,180]$, and $[0,180]$ (resp. $[-100,100]$ ). The similarities between the points of $S_{\text {fixed }}$ and $S_{\text {moving }}$ are computed over a cost matrix, $C_{\text {hist }}$, defined with the $\chi^{2}$ test statistic:

$C_{\text {hist }}\left(p_{i}, q_{j}\right)=\frac{1}{2} \sum_{b=1}^{N b i n s} \frac{\left[h_{i}(b)-h_{j}(b)\right]^{2}}{h_{i}(b)+h_{j}(b)}$

where $h_{i}(b)\left(\right.$ resp. $\left.h_{j}(b)\right)$ represents the bin $b$ of the histogram of point $p_{i}$ (resp. $q_{j}$ ) of $S_{\text {fixed }}$ (resp. $S_{\text {moving }}$ ).

The geodesic cost matrix $C_{\text {geo }}$ is also computed:

$C_{\text {geo }}\left(p_{i}, q_{j}\right)=\left|\operatorname{GeoD}_{\text {fixed }}\left(p_{i}\right)-\operatorname{GeoD}_{\text {moving }}\left(q_{j}\right)\right|$

where $G e o D_{\text {fixed }}\left(p_{i}\right)$ (resp. $G e o D_{\text {moving }}\left(q_{j}\right)$ ) represents the geodesic distance between $p_{i}$ (resp. $q_{j}$ ) and the centroid of the base of $S_{\text {fixed }}$ (resp. $S_{\text {moving }}$ ).

A global cost matrix $C$ is computed with the two weighted normalized cost matrices:

$C_{\varphi_{\text {norm }}}\left(p_{i}, q_{j}\right)=\frac{C_{\varphi}\left(p_{i}, q_{j}\right)-\min \left[C_{\varphi}\left(p_{i}, q_{j}\right)\right]}{\max \left[C_{\varphi}\left(p_{i}, q_{j}\right)\right]-\min \left[c_{\varphi}\left(p_{i}, q_{j}\right)\right]}$

where $\varphi$ is either hist or geo to compute Chist $t_{\text {norm }}\left(p_{i}, q_{j}\right)$ or

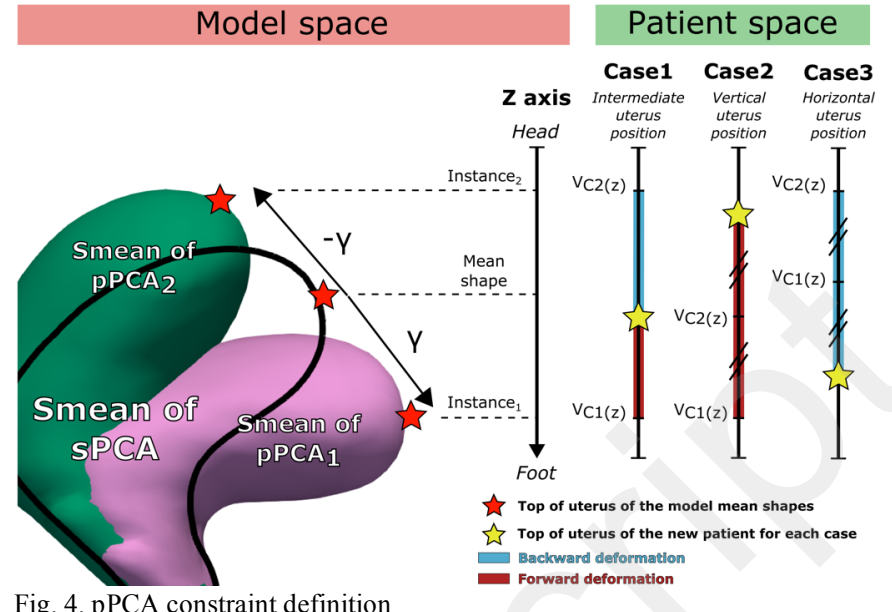

Fig. 4. pPCA constraint definition

The decision process involved in computing the pPCA mean shapes (Smean) of the new patient is illustrated for different cases. $\mathrm{V}_{\mathrm{C} 1}$ and $\mathrm{V}_{\mathrm{C} 2}$ represent the resulting constraint vectors. The constraint vectors length and direction are dependent of the considered case. The cases are explained section II.E.2. The z-axis is pointing to the foot direction.

sPCA: standard principal component analysis; pPCA: posterior PCA

$\operatorname{Cgeo}_{\text {norm }}\left(p_{i}, q_{j}\right)$, respectively.

$C\left(p_{i}, q_{j}\right)=\alpha \times C_{\text {hist }_{\text {norm }}}\left(p_{i}, q_{j}\right)$
$+(1-\alpha) \times C_{\text {geonorm }}\left(p_{i}, q_{j}\right)$

$\alpha \in[0,1]$

To obtain the point-to-point correspondences between $S_{\text {fixed }}$ and $S_{\text {moving }}$, the Hungarian optimization method was used on the $C$ matrix [25]. The correspondences represent the vectors between $S_{\text {fixed }}$ and $S_{\text {moving, }}$, with the minimal cost value in terms of the histogram and geodesic descriptors. Similar to the method implemented by Xiao et al [18], in order to reduce the sensitivity of the shape descriptors, the correspondences that did not respect the following topological constraint were removed:

$$
\begin{aligned}
& \left|\sum_{h=1}^{\text {Nneighb }} D\left(p_{i}, p_{h}\right)-\sum_{h=1}^{\text {Nneighb }} D\left[T\left(p_{i}\right), T\left(p_{h}\right)\right]\right|<\tau \times \\
& \sum_{h=1}^{\text {Nneighb }} D\left(p_{i}, p_{h}\right)
\end{aligned}
$$

where $D\left(p_{i}, p_{h}\right)$ (resp. $\left.D\left[T\left(p_{i}\right), T\left(p_{h}\right)\right]\right)$ represents the Euclidean distance between the tested point $p_{i}$ and its neighbor $p_{h}$ (resp. the correspondences of the tested point $p_{i}$ and of its neighbor $p_{h}$ ), over a total of Nneigh $b=8$ points. This constraint enables the avoidance of large matching irregularities. The parameter $\tau$ is used as a relaxation factor to enable an increase in the number of correspondences driving the deformations.

During the iterative process, $S_{\text {warped }}$ becomes progressively closer to $S_{\text {fixed }}$, thus, the number of correspondences, as well as the non-linearity of the DVF, increase during the process. To improve the optimization, a multiresolution scheme is used that considers different factors of the Taubin filter [26]. For each resolution, Gaussian sigma and topological constraint thresholds $(\tau)$ can be defined. The process is stopped if the mean correspondence cost is greater than the previous one or if the $D V F_{\text {step }}$ mean displacement difference between two iterations is less than a convergence threshold. 


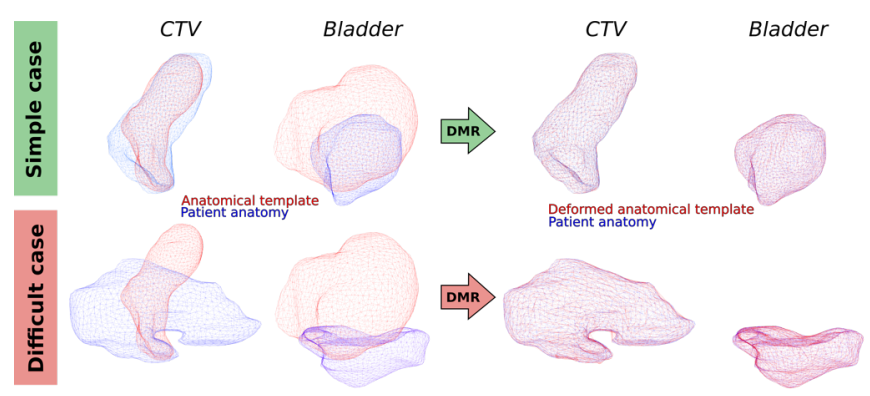

Fig. 5. DMR examples

Two different cases are illustrated. One simple case with similar CTV shapes and small deformations (except for the volume of the bladder) and one difficult case with different CTV shapes and large deformations. In both cases, the proposed DMR method generates a deformation vector field that warps the template shapes to the target patient shapes.

CTV: Clinical target volume; DMR: deformable mesh registration

\section{Standard Principal Component Analysis}

Standard PCA (sPCA) enables the compression of large observations and the organization of the dominant information $[27,28]$. Most studies expressed the dominant deformations of an organ by a compact sPCA model based on few eigenmodes. New samples can then be generated by a weighted sum of these eigenmodes $[29,30]$. Following this, the generated shapes representing the dominant deformations can be combined to define an ITV [31] or to propose a model-generated PTV [32]. Recent studies used the ability of the model to generate random or systematic deformations prior to the treatment according to the training set observation. For example, random treatment planning scenarios have been generated to assess the dose coverage probability in cervical cancer [12]. The patientspecific per-treatment bladder deformations have been predicted in a prostate cancer population [14]. Using early pertreatment images of a patient, regularized PCA-based methods have also been proposed to anticipate systematic deformations [33].

PCA-based models have, thus, been widely used for different applications; however, no study has proposed the use of PCA models to anticipate the clinical target deformations of a new patient in the emergent plan-of-the-day strategy for adaptive RT.

sPCA was used to build a SSM and observe the dominant deformations of the population. A mean shape $\left(S_{\text {mean }}\right)$ was computed from the Nshapes input shapes $\left(S_{i}\right)$ :

$S_{\text {mean }}=\frac{1}{\text { Nshapes }} \sum_{i=1}^{\text {Nshapes }} S_{i}$

The $S_{i}$ shapes represent the inter- and intra-patient CTVs and bladders deformed toward the template anatomy by the DMR. The covariance matrix was computed to represent the linear combination of the shapes:

$\Sigma=\frac{1}{\text { Nshapes }} \sum_{i=1}^{N \text { shapes }}\left(S_{\text {mean }}-S_{i}\right)\left(S_{\text {mean }}-S_{i}\right)^{T}$

The covariance matrix was decomposed to extract the eigenvectors and the eigenvalues, which represent the dominant deformations and their variance, respectively:

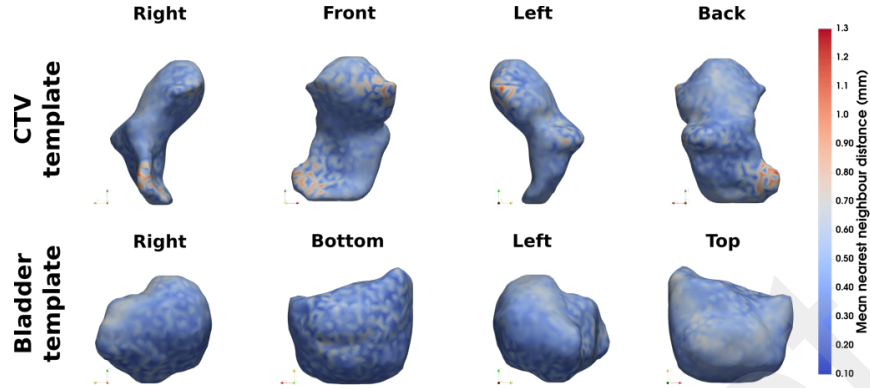

Fig. 6. Illustration of DMR MNND on the template shapes

The MNND is illustrated on the anatomical template showing larger error on the salient local regions of the CTV and larger error on the top part of the bladder.

CTV: Clinical target volume, MNND: mean nearest neighbour distance, DMR: deformable mesh registration

$\Sigma=U D^{2} U^{T}$

where $U$ and $D^{2}$ represent the orthogonal eigenvectors and the corresponding eigenvalues, respectively. Each of the $L$ modes can be scanned independently according to their variance (eigenvalue $\lambda_{1}$ ) in a range of $\pm 3 \sigma$, from the larger deformations to the noisier.

Any shape $S_{\eta}$ can be expressed by the ranked model of $l$ modes $(l<L)$ with the following linear combination, following a normal law $\left(\mathcal{N}\left(S_{\text {mean }}, \Sigma\right)\right)$ :

$S_{\eta}=S_{\text {mean }}+U D_{l}$

Every generated shape $S_{\eta}$ has the same point indices than $S_{\text {mean }}$, thus, a DVF can be computed by the difference of their coordinates.

\section{E. Patient Specific Modeled Planning Library}

The SSM, built by the sPCA over the population, was used to generate potential anatomies for a new patient, based on a single observation. For this purpose, posterior PCA were computed to apply constraints (displacement of the ToU) on the planning anatomy of the new patient. The resulting deformations were applied on this anatomy to build the modeled library, prior to treatment.

\section{1) Posterior Principal Component Analysis}

SSM has been used for shape reconstructions from partial information [34, 35]. The SSM was fitted on the known part of the shape and, thus, the missing part of the shape was reconstructed according to the instance of the model. Furthermore, posterior PCA has been introduced to represent a subset of sPCA with constraints on the known part of a given shape [36].

From the compact sPCA model $\left(S_{\text {mean }}, U D_{l}\right)$, a pPCA model was generated based on a given constraint, $C_{g}$. The resulting pPCA, using Gaussian regression, represents the $S_{C g}$ mean shape and the submatrix $U_{C g} D_{C g}$. The $\mathrm{S}_{\mathrm{Cg}}$ represents a new shape, that satisfies the local constraint $C_{g}$ and with its remaining points interpolated. The submatrix $U_{C g} D_{C g}$ represents the residual eigenvectors and the corresponding 
TABLE I

\begin{tabular}{|c|c|c|c|c|}
\hline \multirow{3}{*}{ Metric } & \multicolumn{4}{|c|}{ Methods } \\
\hline & \multicolumn{2}{|c|}{$\mathrm{RR}$} & \multicolumn{2}{|c|}{ DMR } \\
\hline & CTV & Bladder & CTV & Bladder \\
\hline $\begin{array}{l}\text { MNND } \\
(\mathrm{mm})\end{array}$ & $\begin{array}{c}12.0 \\
(5.5-22.3)\end{array}$ & $\begin{array}{c}10.0 \\
(6.6-19.2)\end{array}$ & $\begin{array}{c}0.5 * \\
(0.3-0.7)\end{array}$ & $\begin{array}{c}0.5 * \\
(0.3-0.8)\end{array}$ \\
\hline $\begin{array}{c}\mathrm{HD} \\
(\mathrm{mm})\end{array}$ & $\begin{array}{c}41.1 \\
(19.0-64.6)\end{array}$ & $\begin{array}{c}51.7 \\
(31.2-70.6)\end{array}$ & $\begin{array}{c}3.3 * \\
(2.5-4.2)\end{array}$ & $\begin{array}{c}3.1 * \\
(2.1-4.4)\end{array}$ \\
\hline DSC & $\begin{array}{c}0.39 \\
(0.22-0.67)\end{array}$ & $\begin{array}{c}0.39 \\
(0.16-0.67)\end{array}$ & $\begin{array}{c}0.98^{*} \\
(0.94-0.99)\end{array}$ & $\begin{array}{c}0.99 * \\
(0.97-1.0)\end{array}$ \\
\hline $\operatorname{ICE}(\mathrm{mm})$ & I & 1 & $5.7(3.2-7.7)$ & $3.8(1.9-6.3)$ \\
\hline
\end{tabular}

The table represents the mean ( $\min -\max )$ values over all patients for each method and organ. * indicates a p-value $<0.001$ for the Wilcoxon test comparing DMR with RR. MNND: Mean nearest neighbor distance; HD: Hausdorff distance; DSC: Dice similarity coefficient; ICE: inverse consistency error; CTV: Clinical target volume

eigenvalues, which are not related to $C_{g}$. To express the uncertainty of $C_{g}$, a Gaussian noise $(\varepsilon)$ is added.

The pPCA formula to express any shape $S_{\eta}$, following a normal law $\left(\mathcal{N}\left(S_{C g}, \Sigma_{C g}\right)\right)$, is then:

$S_{\eta}=S_{C g}+\left(U_{C g} D_{C g}\right)_{l}+\varepsilon$

$\varepsilon \sim \mathcal{N}\left(0, \sigma^{2}\right)$

More detailed calculus and justifications are present in the study conducted by the Scalismo team [36], an open-access library for statistical shape analysis used for this study.

\section{2) Generation of the patient-specific constraint}

To generate two new shapes from the new patient's anatomy using the pPCA, two constraints have to be defined. They were made of two vectors ( $V_{C 1}$ and $V_{C 2}$ in $\mathrm{mm}$ ) applied to the ToU. The ToU point was automatically localized using the geodesic distance to the base centroid (cf. section II.B., eq. (1)). Each constraint was defined according to the signed z-axis distance between the ToU positions of the new patient $\left(\mathrm{ToU}_{\text {new }}\right)$ and of the mean shape of the sPCA (ToU $\mathrm{Smean}_{\text {): }}$

$D=T o U_{\text {new }}(z)-T o U_{S_{\text {mean }}}(z)$

The CTV deformations were analyzed, inside the sPCA space, by a clinical expert to define the mean cervix-uterus shape in a vertical (head direction) and horizontal position (foot direction). From this analysis, the resulting constraint amplitude

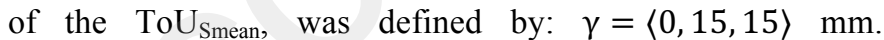
Considering the position of $\mathrm{ToU}_{\text {new }}$ relatively to $\mathrm{ToU}_{\text {Smean }}$, three cases were considered, based on $D$, to generate $V_{C I}$ and $V_{C 2}(c f$. Fig. 4):

$$
\begin{aligned}
& \text { - } \text { Case } 1, \text { if } \frac{-\gamma(\mathrm{z})}{2}<D<\frac{\gamma(\mathrm{z})}{2} \\
& \text { then }\left\{\begin{array}{c}
V_{C 1}(x, y, z)=\langle 0, \gamma(\mathrm{y}), \gamma(\mathrm{z})-D\rangle \\
V_{C 2}(x, y, z)=\langle 0,-\gamma(\mathrm{y}),-\gamma(\mathrm{z})-D\rangle
\end{array}\right.
\end{aligned}
$$

- $\quad$ Case 2 , if $D<\frac{-\gamma(\mathrm{z})}{2}$

$$
\text { then }\left\{\begin{array}{c}
V_{C 1}(x, y, z)=\langle 0, \gamma(\mathrm{y}), \gamma(\mathrm{z})-D\rangle \\
V_{C 2}(x, y, z)=\frac{V_{C 1}(x, y, z)}{2}
\end{array}\right.
$$

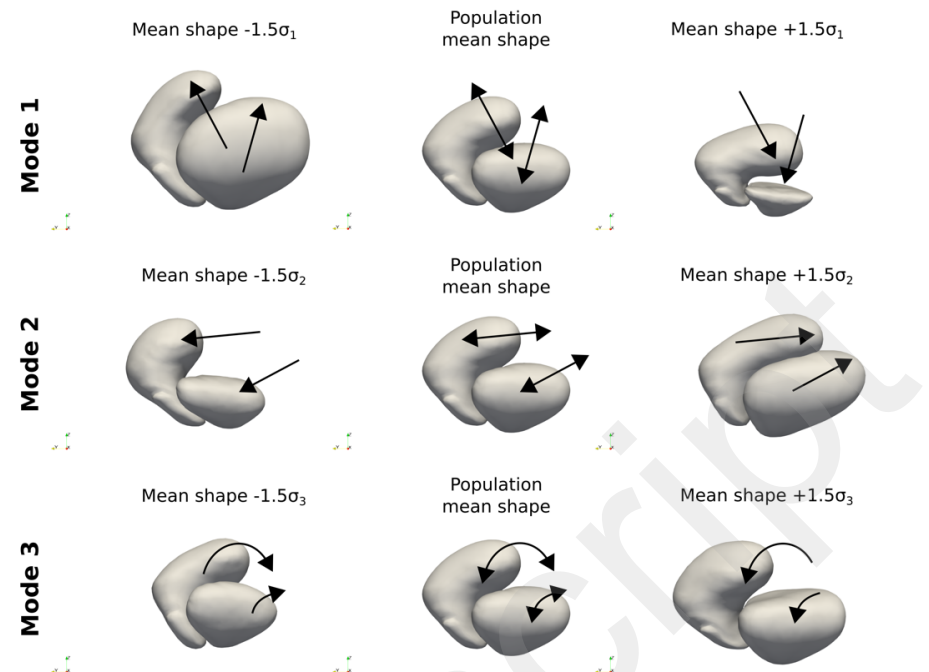

Fig. 7. Three main modes of deformation of the standard principal components analysis

The three main modes of deformation of the cervix-uterus/bladder population SPCA are represented. Mode 1 can be interpreted as a superior/inferior deformation of the uterus with a large variation in the bladder volume. Modes 2 and 3 correspond to an ante-posterior shrinking and a leftright rotation on the $\mathrm{Y}$-axis of the anatomical structures, respectively.

PCA: principal component analysis; $\sigma_{\mathrm{x}}^{2}$ : variance of the $\mathrm{x}^{\text {th }}$ mode (eigenvalue)

$$
\begin{aligned}
& \text { - } \quad \text { Case } 3 \text {, if } D>\frac{\gamma(\mathrm{z})}{2} \\
& \text { then }\left\{\begin{array}{c}
V_{C 2}(x, y, z)=\langle 0,-\gamma(\mathrm{y}),-\gamma(\mathrm{z})-D\rangle \\
V_{C 1}(x, y, z)=\frac{V_{C 2}(x, y, z)}{2}
\end{array}\right.
\end{aligned}
$$

The two defined constraints enabled the computation of two pPCA mean shapes and their eigenvectors (cf. section II.E.1). The two DVFs between each pPCA and the sPCA on the CTV and bladder mean shapes were extracted. Finally, the new patient's planning CTV and bladder were deformed by these DVFs.

Due to the difference in the bladder shapes and volumes among the patients, a basic representation of the bladder mean shape of the population may lead to unsatisfying bladder DVFs. Indeed, the initial bladder of the patient may be larger or smaller than the population mean bladder. Thus, the bladder DVF from the model can be improper in term of amplitude (i.e., resulting in uterus-bladder overlap for large initial bladder or unrealistic deformations for small initial bladder). To correct this, a ratio was applied on the DVF amplitude corresponding to the difference between the position of the top of the bladder of each shape (patient and sPCA bladder shapes). Moreover, the deformed bladder was iteratively smoothed and deflated, where the local uterus-bladder overlap occurs, following the inverse of the vertex normal.

After the denormalization of the deformed shapes, the pretreatment library, containing the two modeled CTVs and bladders shapes and the patient original planning CT anatomy, was obtained.

\section{EVALUATION}

\section{A. Dataset}

The method was evaluated on a total of 20 subjects. Each underwent three planning CTs with different bladder volumes 


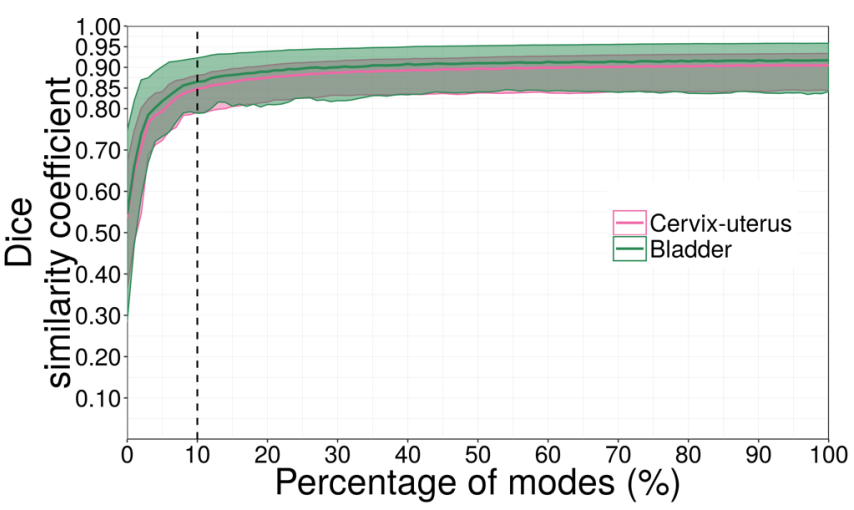

(a)

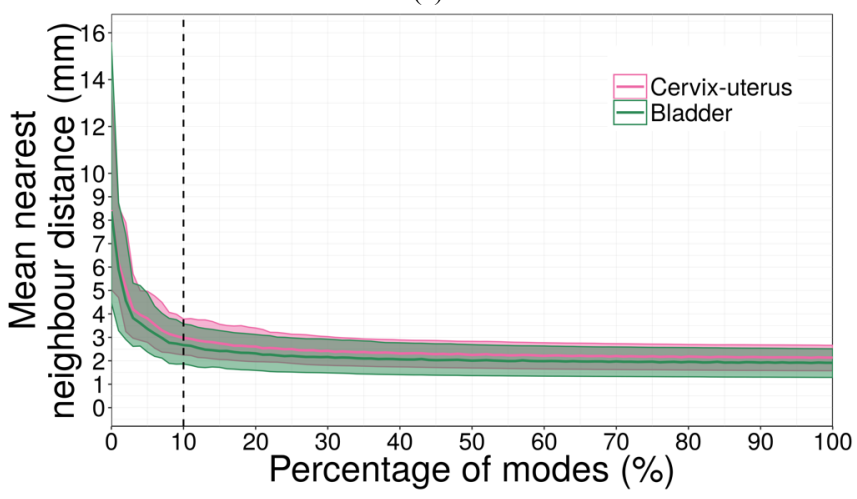

(b)

Fig. 8. Standard PCA performance of the two shapes in all ranks by considering: (a) the DSC and (b) the MNND

The multi-organ PCA performances are illustrated for all tested modes using leave-one-patient-out cross-validation.

PCA: Principal component analysis; DSC: Dice similarity coefficient; MNND: Mean nearest neighbour distance

(empty, intermediate, and full) and, at least, bi-weekly CBCTs during the 5 weeks of treatment (total 314 images). The CTV, including the cervix and uterus, and the OARs (rectum, bladder, and bowel) were delineated by the same expert on $\mathrm{CT}$ and CBCT images. The surfaces of the delineations were smoothed using Taubin filter [26], decimated and remeshed using the approximated centroidal Voronoi diagrams (ACVD) method [37], resulting in smooth meshes of 1500 equidistant points.

The experiments were performed following a leave-onepatient-out cross-validation. A PCA-based model was defined for each patient using the 19 other patients for the training set.

Considering the iterative DMR, the following parameters were empirically selected: 3 resolutions of smoothing (Taubin filter passband $=[0.01,0.1,1])$; cost matrix weight factor $\alpha=0.5$; Gaussian sigma $\sigma=[4,2,1]$; step update factor $\mu=0.25$; and topology constraint threshold $\tau=[0.25,0.5,1]$.

In order to keep the dominant information from the training set and to filter out the noisy deformations (i.e., resulting from DMR uncertainties or intra-observer delineation variations), a rank threshold of $10 \%$ was therefore considered for the sPCA (cf. section III.E.). Thus, the pPCA models were defined on $10 \%$ of the sPCA first modes.

The proposed DMR method was developed using the VTK library. Statistical shape analysis was performed using the Scalismo library. A non-parametric test (paired Wilcoxon test) was used to compare the performance of the strategies and to assess if a strategy was statistically different from another. The $\mathrm{R}$ software was used for this purpose.

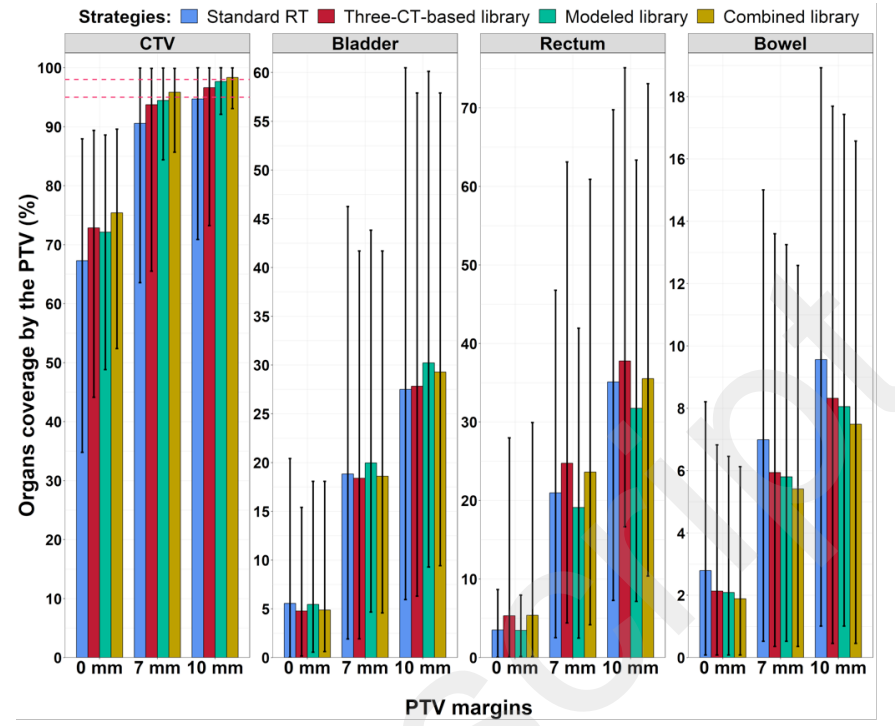

Fig. 9. CTV and OAR coverage by the PTV with various margins for all strategies

The colors represent the RT strategies, and the x-axis represents the PTV margins $(0,7$, and $10 \mathrm{~mm})$. The filled bars represent the mean values of the population (with one value per patient, corresponding to the mean coverage over the treatment), and the black lines represent the min and max values. The optimal treatment corresponds to a high CTV coverage and a low OAR coverage. The red dashed lines represent $95 \%$ and $98 \%$ CTV coverage by the PTV, respectively.

CTV: Clinical target volume; OAR: Organs at risk (bladder, rectum and bowel); PTV: Planning target volume; RT: Radiation therapy

\section{B. Simulation of Treatment Delivery}

As considered in clinical routine, for each CTV (either resulting from the CTs segmentation or from the model), a PTV was generated by adding an isotropic margin. To simulate patient positioning during treatment delivery, all the CBCTs were bone-registered with the planning $\mathrm{CT}$ (intermediate bladder). For the standard RT, only the PTV with intermediate bladder volume was used to simulate the treatment. For the library-based strategies, multiple PTV ( 3 or 5, $c f$. Fig. 1) were available for each treatment fraction. The PTV selected for each CBCT was the one providing the maximum coverage with the CTV delineated on the CBCT. In the event of equal overlap by multiple PTVs, the PTV with the lowest volume was selected.

The modeled planning library was evaluated and compared against the standard RT (based on the intermediate bladder volume CT) and the three-CT-based planning library, including, for each patient, the shapes of the three planning CTs (empty, intermediate and full bladder volumes). A combined library, including the three-CT-based and modeled library shapes, totaling 5 shapes, was also evaluated ( $c f$. Fig 1d).

\section{Evaluation of the Treatment Strategies}

The criterion used to assess the best strategy was the coverage of the volumes of interest (CTV and OAR) by the PTV. The "coverage of the organ by the PTV" was computed as the intersection between the selected PTV and the structure delineation, normalized according to the structure volume. This coverage criterion represented the ability of the considered strategy to cover the target or to spare the organs at risk for each 


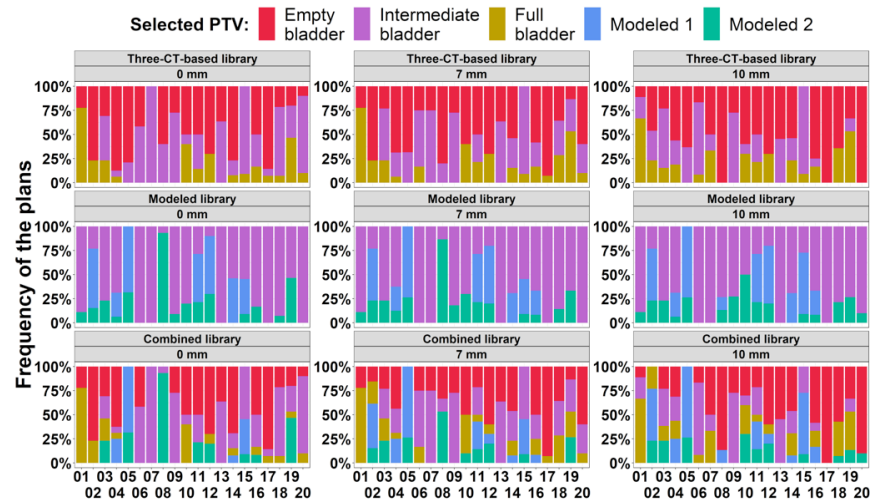

Fig. 10. Selected PTV during the treatment of each patient.

The y-axis represents the frequency of the PTV selected during the treatment simulations. The colors represent the different PTV. The $\mathrm{x}$-axis represents the patient identifiers. Each facet represents a strategy with a PTV margin $(0,7$, and $10 \mathrm{~mm})$. PTV: Planning target volume;

treatment fraction and, thus, of its ability to anticipate the deformations. For each patient, the coverages of the volumes of interest were averaged by the number of fractions (i.e., CBCTs) to represent the whole treatment.

All the strategies were tested with $0 \mathrm{~mm}, 7 \mathrm{~mm}$ and $10 \mathrm{~mm}$ PTV margins (i.e., isotropic dilatation of the CTV to generate the PTV). The $0 \mathrm{~mm}$ PTV margin, if clinically unrealistic, was considered to assess the coverage of the organs provided by the geometrical model only.

\section{Deformable Mesh Registration Performance}

Fig. 5 illustrates two cases of estimated deformation, namely simple and difficult, based on the similarity between the template and a patient.

Fig. 6 represents the mean nearest neighbor distance (MNND) on the template shapes after DMR. The largest errors were located on the salient local regions of the CTV and on the top part of the bladder.

Table 1 represents the rigid registration (RR, section II.B.), and DMR performances using the following metrics: MNND, Hausdorff distance (HD), Dice similarity coefficient (DSC) and inverse consistency error (ICE). Compared to the RR, the DMR performances were significantly better.

\section{E. Principal Component Analysis}

The sPCA-based model was cross-validated using a leaveone-patient-out procedure across the 20 subjects.

Fig. 7 represents the three main modes of deformation of the cervix-uterus/bladder sPCA. The first mode can be interpreted as the top of the uterus displacement according to the bladder filling. The second and third modes correspond to an anteposterior shrinking and a left-right rotation on the Y-axis of the shapes, respectively.

Fig. 8ab represents the DSC and MNND for each organ as a function of the SPCA modes, and thus, the ability of the SPCA to explain the inter- and intra-patient variation with a given number of modes. The Fig. 8ab illustrates, thus, the generalization ability of the models to represent unseen shapes in the training set (i.e., following leave-one-patient-out cross validation) [38]. At a $10 \%$ rank, the DSC and MNND were 0.85 $(0.79-0.88)$ and $3.0 \mathrm{~mm}(2.2-3.8)$ for the cervix-uterus and
$0.86(0.79-0.92)$ and $2.7 \mathrm{~mm}(1.9-3.6)$ for the bladder, respectively. At this rank, $90 \%$ of the maximum DSC was reached for each organ.

\section{F. Modeled library}

Fig. 9 represents the strategy performances in terms of the CTV and OAR coverage by the PTV, for each margin value.

Considering the CTV coverage by the PTV, compared to the standard RT, all strategies significantly increased the coverage $(\mathrm{p}<0.001)$. Compared the standard RT, the modeled library was beneficial for $75 \%$ of the patients (with no impact for the other). The modeled library had similar performances compared to the three-CT-based library $(\mathrm{p}=0.95)$.

Importantly, considering a $10 \mathrm{~mm}$ PTV margin, the minimal value of the CTV coverage increased from $73.2 \%$ with the three-CT-based library to $92.1 \%$ with the modeled libraries. Also, the combined library significantly increased the CTV coverage, compared to all strategies: $+3.6 \%$ compared to the standard RT $(\mathrm{p}<0.001) ;+1.7 \%$ compared to the three-CT-based library $(\mathrm{p}<0.001)$ and $+0.7 \%$ compared to the modeled library $(\mathrm{p}<0.001)$.

Comparing the modeled library with the classic library at 10 $\mathrm{mm}$ PTV margin, $20 \%$ of the patients had an increase of CTV coverage superior to $1 \%$ (up to $21.3 \%$ ) while $10 \%$ had a decrease superior to $1 \%$ (up to $4 \%$ ). By combining the two libraries, $25 \%$ of the patients had an increase of the CTV coverage superior to $1 \%$ (up to $21.3 \%$ ) and none had a decrease.

Considering the OAR coverage by the PTV, the modeled library significantly increased the sparing of the rectum $(-6 \%$, $\mathrm{p}<0.001)$ compared to the three-CT-based library. The modeled and combined libraries increased the sparing of the bowel ($0.3 \%$ and $-0.8 \%$ [eq. $1.2 \mathrm{cc}$ and $2.4 \mathrm{cc}$ ], $\mathrm{p}=0.08$ and $\mathrm{p}<0.001$ ). However, the modeled and combined library significantly overcovered the bladder $(+2.4 \%$ and $+1.5 \%$; $p<0.001)$.

Considering the impact of the PTV margins on CTV coverage, an improvement of approximately $25 \%$ was observed by increasing the margin from 0 to $7 \mathrm{~mm}$ and of $5 \%$ from 7 to $10 \mathrm{~mm}$. Concerning the impact of the PTV margins on the OAR, an impairment of $15 \%$ was observed by increasing the margin from 0 to $7 \mathrm{~mm}$ and of $10 \%$ from 7 to $10 \mathrm{~mm}$ for the rectum and bladder. In total, if the use of adaptive strategies will enable to reduce the margins, their choice remains crucial.

Fig. 10 represents the frequency of fractions for which the plans were used, according to the strategy and for each individual. Considering the modeled library, the modeled plans were used at least once for $75 \%$ of the patients. Considering all the available plans using the combined library, the modeled plans were used at least once for $60 \%$ of the patients. 


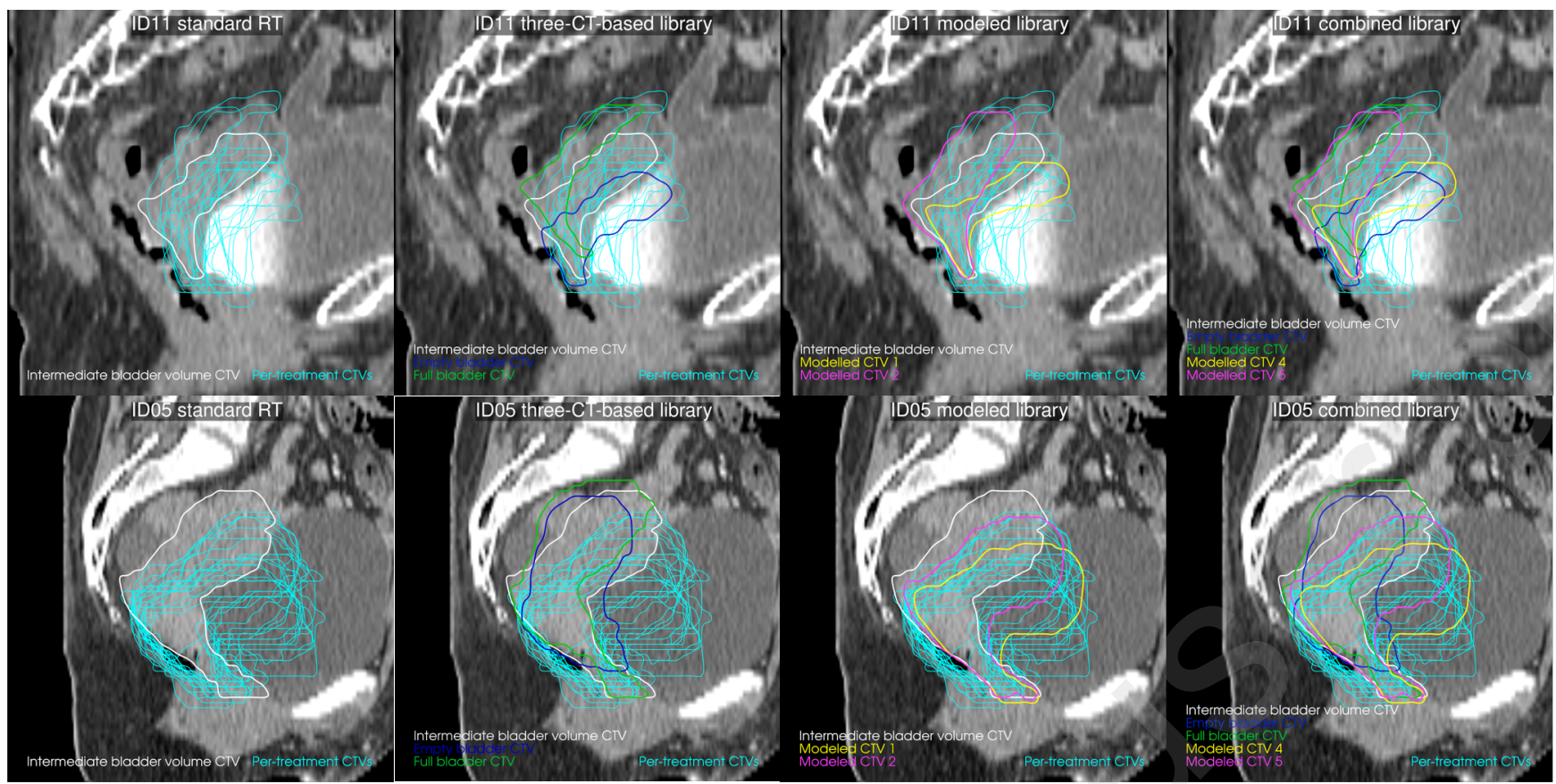

Fig. 11. Example of the RT strategies used for two different patients

The patient on the first row has a moving uterus prior to and during the treatment. The patient on the second row has a non-moving uterus prior to the treatment

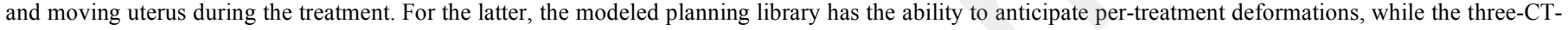

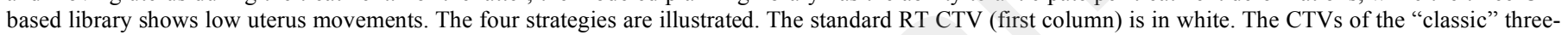
CT-based library (second column) are in white, dark blue and green. The CTVs of the patient-specific modeled library (third column) are in white, pink and yellow. The CTVs of the combined library (fourth column) are in white, dark blue, green, yellow and pink. The per-treatment CTV contours are in light blue.

In this particular order, the CTV coverages by the $10 \mathrm{~mm}$ PTV of the patient of the first row were $84.8 \%, 92.0 \%, 92.1 \%$ and $93.1 \%$. The CTV coverages by the $10 \mathrm{~mm}$ PTV of the patient of the second row were $70.9 \%, 73.2 \%, 94.5 \%$ and $94.5 \%$.

CTV: Clinical target volume

Fig. 11 illustrates each strategy for two different patients: the first one (upper row), had a moving uterus at the planning and during the treatment; the second one had a non-moving uterus at the planning, which became mover during the treatment. For the first patient, both libraries performed similarly. However, for the second patient, the modeled library better anticipated the deformations than the three-CT-based library. Indeed, before treatment, the tumor may prevent the uterus from moving, whereas during treatment, the tumor shrinks and then lets the uterus move.

In terms of computation time, the proposed approach simulates the two modeled CTs in less than 3 minutes per patient (approximately 1 minute per organ for the DMR and 1 minute for the pPCA-based models). Compared to the threeCT-based library, only the combined library would increase the workload in term of treatment plan optimization.

\section{DISCUSSION AND CONCLUSION}

We have proposed a workflow that models a planning library by taking into account population deformations. This workflow relies on two steps. First, a DMR method was proposed to compute the inter-patient cervix-uterus and bladder correspondences. Second, a SSM was built to deform the specific-patient anatomy to generate a modeled library.

This study has several limitations. First, the DMR performance was only evaluated considering geometric metrics and not using fiducial markers or manually placed landmarks. Landmark positioning appears, however, difficult on pelvic anatomy, and a numerical phantom could have been used as reported when considering prostate cancer [39]. The ICE were larger than the ones reported in the literature using symmetric registration methods $[12,20]$ due to the fact that our method computes the DVF in only one direction (i.e., forward). Still, the proposed method provides the smallest reported ICE of the non-symmetrical registration method [20] (cf. Table 1).

Second, the statistical shape analysis was done by mixing both intra- and inter-patient deformations, without taking the patient's deformations among the population specifically into account. One proposed solution would be to consider the interfractions average shape per patient [40]. To overcome this shortcoming, the population was normalized according to a common space, limiting the deformation analysis on the uterus. Moreover, the model deformation vector fields were applied on the specific patient's planning anatomy.

Finally, the modeled planning library performance was assessed using only a geometric criterion, instead of dosimetric criteria that are more important in a clinical perspective. The workload implied by dose optimization and calculation prevent the exhaustive dosimetric evaluation of new strategies. Moreover, a previous study has shown a good agreement between geometric and dosimetric criteria for cervical cancer patients [8]. In the end, the clinical benefits of such complex strategies will have to be evaluated on a larger cohort.

The modeled and combined libraries performed similarly or better than a standard RT or classic CT-based library. In particular, the coverage of the target was largely improved for patients with non-moving CTV at the planning. Moreover, the proposed modeled approach reduces the need for the heavy three-CT-acquisition protocol. The use of the proposed 
combined library, including the modeled shapes, would increase the workload of the physicist at the planning ( $2 \mathrm{~h}$ per plan optimization). However, the need for adaptive strategies, based on the generation of multiple treatment plans, is largely accepted and some clinical tools, such as automatic planning tools [41], may ease their clinical implementation. Two ongoing clinical trials based on the use of the planning library showed that 3 to $15 \mathrm{~min}$ is required to select the plan-of-the-day at each treatment fraction $[10,42]$.

The perspective of this work is to compute planning doses with the modeled shapes on a water-air-bone pseudo-CT to dosimetrically confirm the results. The proposed method can be derived to evaluate similar adaptive strategies for bladder and rectal cancers.

\section{REFERENCES}

[1] R. Jadon, C. Pembroke, C. Hanna et al., "A systematic review of organ motion and image-guided strategies in external beam radiotherapy for cervical cancer," Clinical Oncology, vol. 26, no. 4, pp. 185-196, 2014.

[2] R. Ahmad, M. S. Hoogeman, M. Bondar et al., "Increasing treatment accuracy for cervical cancer patients using correlations between bladder-filling change and cervix-uterus displacements: proof of principle," Radiotherapy and oncology, vol. 98, no. 3, pp. 340-346, 2011.

[3] Y. Han, E. H. Shin, S. J. Huh et al., "Interfractional dose variation during intensity-modulated radiation therapy for cervical cancer assessed by weekly CT evaluation," International Journal of Radiation Oncology* Biology* Physics, vol. 65, no. 2, pp. 617623, 2006.

[4] A. Buchali, S. Koswig, S. Dinges et al., "Impact of the filling status of the bladder and rectum on their integral dose distribution and the movement of the uterus in the treatment planning of gynaecological cancer," Radiotherapy and oncology, vol. 52, no. 1, pp. 29-34, 1999.

[5] L. van de Bunt, I. M. Jürgenliemk-Schulz, G. A. de Kort et al., "Motion and deformation of the target volumes during IMRT for cervical cancer: what margins do we need?," Radiotherapy and Oncology, vol. 88, no. 2, pp. 233-240, 2008.

[6] Y. Seppenwoolde, M. Stock, M. Buschmann et al., "Impact of organ shape variations on margin concepts for cervix cancer ART," Radiotherapy and Oncology, 2016.

[7] M. Bondar, M. Hoogeman, J. Mens et al., "Individualized nonadaptive and online-adaptive intensity-modulated radiotherapy treatment strategies for cervical cancer patients based on pretreatment acquired variable bladder filling computed tomography scans," International Journal of Radiation Oncology* Biology* Physics, vol. 83, no. 5, pp. 1617-1623, 2012.

[8] B. Rigaud, A. Simon, M. Gobeli et al., "CBCT-guided evolutive library for cervical adaptive IMRT," Medical Physics, 2018.
M. Gobeli, A. Simon, M. Getain et al., "Benefit of a pretreatment planning library-based adaptive radiotherapy for cervix carcinoma?," Cancer radiotherapie, vol. 19, no. 6-7, pp. 471-478, 2015.

S. T. Heijkoop, T. R. Langerak, S. Quint et al., "Clinical implementation of an online adaptive planof-the-day protocol for nonrigid motion management in locally advanced cervical cancer IMRT," Int. J. Radiat. Oncol. Biol. Phys., vol. 90, no. 3, pp. 673-679, 2014.

A. J. van de Schoot, P. de Boer, J. Visser et al., "Dosimetric advantages of a clinical daily adaptive plan selection strategy compared with a non-adaptive strategy in cervical cancer radiation therapy," Acta Oncologica, vol. 56, no. 5, pp. 667-674, 2017.

D. Tilly, A. J. van de Schoot, E. Grusell et al., "Dose coverage calculation using a statistical shape modelapplied to cervical cancer radiotherapy," Physics in Medicine and Biology, vol. 62, no. 10, pp. 4140, 2017. L. Tu, M. Styner, J. Vicory et al., "Skeletal Shape Correspondence Through Entropy," IEEE Transactions on Medical Imaging, vol. 37, no. 1, pp. $1-11,2018$.

R. Rios, R. De Crevoisier, J. D. Ospina et al., "Population model of bladder motion and deformation based on dominant eigenmodes and mixed-effects models in prostate cancer radiotherapy," Medical image analysis, vol. 38, pp. 133-149, 2017.

A. Kelemen, G. Székely, and G. Gerig, "Elastic model-based segmentation of 3-D neuroradiological data sets," IEEE Transactions on medical imaging, vol. 18 , no. 10 , pp. 828-839, 1999.

S. Belongie, J. Malik, and J. Puzicha, "Shape matching and object recognition using shape contexts," IEEE transactions on pattern analysis and machine intelligence, vol. 24, no. 4, pp. 509-522, 2002.

O. Acosta, J. Fripp, V. Doré et al., "Cortical surface mapping using topology correction, partial flattening and 3D shape context-based non-rigid registration for use in quantifying atrophy in Alzheimer's disease," Journal of Neuroscience Methods, vol. 205, no. 1, pp. 96-109, 2012.

D. Xiao, D. Zahra, P. Bourgeat et al., "An improved 3D shape context based non-rigid registration method and its application to small animal skeletons registration," Computerized Medical Imaging and Graphics, vol. 34, no. 4, pp. 321-332, 2010.

J. Ruhaak, T. Polzin, S. Heldmann et al., "Estimation of Large Motion in Lung CT by Integrating Regularized Keypoint Correspondences into Dense Deformable Registration," IEEE Transactions on Medical Imaging, 2017.

L. Bondar, M. S. Hoogeman, E. M. V. Osorio et al., "A symmetric nonrigid registration method to handle large organ deformations in cervical cancer patients," Medical physics, vol. 37, no. 7, pp. 3760-3772, 2010. J. Yang, "The thin plate spline robust point matching (TPS-RPM) algorithm: A revisit," Pattern Recognition Letters, vol. 32, no. 7, pp. 910-918, 2011. 
[22] H. Chui, and A. Rangarajan, "A new point matching algorithm for non-rigid registration," Computer Vision and Image Understanding, vol. 89, no. 2, pp. 114-141, 2003.

[23] E. M. Vásquez Osorio, I. K. K. Kolkman- Deurloo, M. Schuring- Pereira et al., "Improving anatomical mapping of complexly deformed anatomy for external beam radiotherapy and brachytherapy dose accumulation in cervical cancer," Medical physics, vol. 42, no. 1, pp. 206-220, 2015.

[24] A. Myronenko, and X. Song, "Point set registration: Coherent point drift," IEEE transactions on pattern analysis and machine intelligence, vol. 32, no. 12, pp. 2262-2275, 2010.

[25] R. Jonker, and A. Volgenant, "A shortest augmenting path algorithm for dense and sparse linear assignment problems," Computing, vol. 38, no. 4, pp. 325-340, 1987.

[26] G. Taubin, T. Zhang, and G. Golub, "Optimal surface smoothing as filter design," Computer VisionECCV'96, pp. 283-292, 1996.

[27] J. E. Jackson, A user's guide to principal components: John Wiley \& Sons, 2005.

[28] J. J. Cerrolaza, R. M. Summers, and M. G. Linguraru, "Soft Multi-organ Shape Models via Generalized PCA: A General Framework." pp. 219-228.

[29] M. Söhn, M. Birkner, D. Yan et al., "Modelling individual geometric variation based on dominant eigenmodes of organ deformation: implementation and evaluation," Physics in Medicine and Biology, vol. 50, no. 24, pp. 5893, 2005.

[30] E. Brion, C. Richterb, B. Macqa et al., "Modeling patterns of anatomical deformations in prostate patients undergoing radiation therapy with an endorectal balloon." pp. 1013506-1013506-9.

[31] S. Thörnqvist, L. B. Hysing, A. G. Zolnay et al., "Adaptive radiotherapy in locally advanced prostate cancer using a statistical deformable motion model," Acta Oncologica, vol. 52, no. 7, pp. 1423-1429, 2013.

[32] L. Bondar, M. Intven, J. M. Burbach et al., "Statistical Modeling of CTV Motion and Deformation for IMRT of Early-Stage Rectal Cancer," International Journal of Radiation Oncology* Biology* Physics, vol. 90, no. 3, pp. 664-672, 2014.

[33] M. A. Chetvertkov, F. Siddiqui, J. Kim et al., "Use of regularized principal component analysis to model anatomical changes during head and neck radiation therapy for treatment adaptation and response assessment," Medical physics, vol. 43, no. 10, pp. 5307-5319, 2016.

[34] S. I. Buchaillard, S. H. Ong, Y. Payan et al., "3D statistical models for tooth surface reconstruction," Computers in Biology and Medicine, vol. 37, no. 10, pp. 1461-1471, 2007.

[35] S. Poltaretskyi, J. Chaoui, M. Mayya et al., "Prediction of the pre-morbid 3D anatomy of the proximal humerus based on statistical shape modelling," Bone Joint J, vol. 99, no. 7, pp. 927-933, 2017.
[36] T. Albrecht, M. Lüthi, T. Gerig et al., "Posterior shape models," Medical image analysis, vol. 17, no. 8, pp. 959-973, 2013.

[37] S. Valette, J. M. Chassery, and R. Prost, "Generic remeshing of $3 \mathrm{D}$ triangular meshes with metricdependent discrete Voronoi diagrams," IEEE Transactions on Visualization and Computer Graphics, vol. 14, no. 2, pp. 369-381, 2008.

[38] M. A. Styner, K. T. Rajamani, L.-P. Nolte et al., "Evaluation of 3D correspondence methods for model building." pp. 63-75.

[39] G. Cazoulat, A. Simon, A. Dumenil et al., "Surfaceconstrained nonrigid registration for dose monitoring in prostate cancer radiotherapy," IEEE Transactions on Medical Imaging, vol. 33, no. 7, pp. 1464-1474, 2014.

[40] E. Budiarto, M. Keijzer, P. Storchi et al., "A population-based model to describe geometrical uncertainties in radiotherapy: applied to prostate cases," Physics in medicine and biology, vol. 56, no. 4, pp. 1045, 2011.

[41] A. W. M. Sharfo, S. Breedveld, P. W. Voet et al., "Validation of fully automated VMAT plan generation for library-based plan-of-the-day cervical cancer radiotherapy," PloS one, vol. 11, no. 12, pp. e0169202, 2016.

[42] M. Buschmann, K. Majercakova, A. Sturdza et al., "Image guided adaptive external beam radiation therapy for cervix cancer: Evaluation of a clinically implemented plan-of-the-day technique," Zeitschrift für Medizinische Physik, 2017. 